By using nuclei of different sizes together with detection in coincidence of the leading hadron in the final state, it is possible to examine two fundamental aspects of the string, namely the formation length of the hadrons and the string's interaction with the nuclear medium.

\section{An Accelerator for Hadronic Physics}

Electron scattering has been used extensively to study quark distributions since the pioneering work nearly 25 years ago at SLAC by this year's Nobel laureates (see page 208) that gave the first evidence for the presence of quarks in the nucleon. In these experiments, one varies independently the momentum and energy transfer to nuclear constituents by adjusting the incident energy and scattering angle of the electrons. Muons and neutrinos are also used but only to a limited extent as the fluxes that can be achieved in practice are considerably less intense (by at least a factor of $10^{6}$ ).

Their exists today a wealth of electron scattering data but it contains very little information on specific final states. Most of the data that is available on hadronic physics is of poor quality and inconclusive because suitable experimental facilities are lacking. Existing high-energy electron accelerators have pulsed beams, where each beam burst leads to electron-nucleus collisions that produce an extremely large instantaneous flux of all kinds of emitted particles which blinds detectors. In order to observe the coincident emission of particles corresponding to final states, it is imperative to decrease the peak current of each beam burst and to maximize the number of bursts (the ideal being a continuous beam).

An additional requirement is a suitable energy range. The physics programmes that were discussed at the Dourdan workshop invariably require incident electron energies of $15-25 \mathrm{GeV}$. For example, a detailed study of the electroproduction of charmed particles at energies below $15 \mathrm{GeV}$ would be rather limited. Similarly, information about hadronization cannot be revealed using high energy studies above $30 \mathrm{GeV}$, where the production of hadrons would occur outside the nucleus. These and other considerations imply an accelerator having the following characteristics: energy $15-25 \mathrm{GeV}$

$$
\begin{aligned}
& \text { energy resolution }<50 \mathrm{MeV} \\
& \text { duty cycle }>50 \% \\
& \text { intensity }>50 \mu \mathrm{A} .
\end{aligned}
$$

Several possible designs for a continuous intermediate energy electron accelerator were presented in the working session "Experimental Facilities" (P. Dalpiaz, J. Domingo, J.L. Faure). Superconducting RF accelerating cavities will be essential and major improvements in their performance were reported, notably by a group of 60 physicists and engineers at the CEN, Saclay which is pushing the frontiers of this technology and is building a prototype of an electron accelerator using new types of cavity design.

Europe has not yet developed a plan for a facility that would compete with US accelerators, notably the $4 \mathrm{GeV}$ CEBAF electron accelerator under construction and the RHIC relativistic heavy ion collider that is included in the 1991 Federal budget. An European machine with the above characteristics would reach beyond CEBAF: its realization appears challenging but feasible in the light of technical developments that are under study.

Several continuous electron beam facilities will soon be available in Europe (at Bonn, Mainz and Amsterdam) for nuclear structure studies in the 1 to $4 \mathrm{GeV}$ range. Meanwhile, preliminary exploration of hadronic physics will continue with particle physics accelerators such as SLAC and HERA, and with muon beams at Fermilab and CERN. Work at these facilities will show physicists how to optimize new detectors and to design the next generation of experiments.

\section{Future Perspectives}

Now that there is a measure of consensus on the scientific goals and key investigations in hadronic physics, the next step is an in-depth study of the experiments that were proposed at the workshop. The aim will be to arrive at more precise estimates of the optimum characteristics for a state-of-the-art continuous beam electron accelerator. The most obvious concern is to design a cost-effective machine that would satisfy the needs of a large users' community. The conclusions of the workshop will be taken up in the meantime by NuPECC, the Nuclear Physics European Coordination Committee (see Europhysics News 21 (1990) 180) in its preparation, starting next year, of a longterm plan for European nuclear physics.

\section{REFERENCES}

[1] Sick I., Europhys. News 21 (1990) 111. [2] Hadronic Physics in the 1990's with MultiGeV Electrons, Nucl. Phys. A497 (1989) 1.

[3] Bialas A. and Czyzewski J., Phys. Lett. 222B (1989) 132

\title{
Designing With Structural Ceramics
}

\author{
EIW-5, Europhysics Industrial Workshop \\ Petten, 3-6 April 1990
}

The Europhysics Industrial Workshop EIW- 5 was held at Petten in the Netherlands in April 1990. Two cooperating organizations, the Netherlands Energy Research Foundation (ECN) and the Commission of the European Communities, Institute of Advanced Materials, JRC-P, were responsible for the organization.

The meeting aimed to present the stateof-the-art of the application of ceramics in technology, to develop insights into directions for future $R \& D$, and to explore the possibilities for further applications of advanced ceramics in industry. For it is absolutely essential that important progress is made to arrive at a situation where the properties of the materials which can be produced are used to their fullest extent in advanced applications. It must emphasized that designers should demonstrate a greater willingness to use ceramics and to design in such a way that these materials will function satisfactorily. However, a number of critical technologies will also have to be developed:

- Fabrication of dense ceramics and composites without critical defects which degrade the mechanical properties.

- New ways to realize mechanical bonds between ceramics and metals or other ceramics.

- New skills in the field of engineering and design to improve reliability.

Although the workshop intended to focus on the last topic, the presentations and discussion stressed the fabrication and characterization of advanced cera- mics. In this respect, it was found that the aerospace industry is of vital importance to stimulate new developments in structural ceramics. Nevertheless, in spite of this tendency, a number of current industrial applications are worth mentioning to illustrate the present situation.

The aerospace industry clearly focusses on the development of high strength, fibre reinforced composite materials that will be accommodated in a new generation of high velocity aircraft like the STS 2000. The requirements imposed on the materials are well defined, but the materials still find themselves in a state of initial development, with the emphasis on advanced ceramics for locations where material costs are not critical.

A further development worth mentioning is the use of ceramics in the automotive industry. A key feature is to improve high-temperature strength and the reliability. One has not yet succeeded in making components which are sufficiently free from defects to guarantee failure-free operation for long periods of time. This fact gives a strong need for design methods based upon the acceptance of defects in a material (see page 215). Defect tolerance can be obviously achieved by good design involving small volumes, constant wall thicknesses and the absence of tensile stresses or stress concentrations (see page 215). At this moment it should be concluded that practical applications of ceramics in the automotive industry are limited to parts subjected to static stres- 
ses, and to thermal insulating coatings. Presently, however, the improvement to more traditional materials, e.g. metals, are such that new ceramics have a serious problem in making themselves acceptable alternatives.

In spite of the generally high level of skills available to make ceramics, to bond them to metals and to achieve well controlled surface finishes, besides the understanding of the behaviour of the materials under service conditions, it can be concluded that the introduction in technological structures intended for mass production is seriously behind schedule. But this should not lead to the conclusion that interest in engineering ceramics is reducing. Defect control and reliability during fabrication deserve more attention, along with pro- moting a willingness to accept materials which still have a lower reliability level than metals. In this respect, the particular advantages of ceramics such as chemical or thermal resistance and wear properties, are presently in an exploratory stage over a broad range of applications. Slightly outside the scope of the workshop, it was emphasized that in the process industry and activities related to energy production and the environment, a number of applications of advanced ceramics are within reach. These applications often involve porous ceramic materials to control processes and fulfill tasks involving separation and clearing.

\section{Veringa ECN Petten}

stress relative to the failure strength of the ceramic.

Fig. 1 also shows the calculated stresses for a prestressed ring in its shrinkage holder after loading: the stresses are compressive and nowhere more than 0.30 times the failure strength. This fairly conservative, perhaps unspectacular, approach clearly works as the company has been using the rings to produce cans at a rate of four each second, where a failure could be disastrous.

Towards An Accurate Statistical Approach So-called weak-link or statistical failure prediction is based upon estimating the strength of a brittle material in terms of a global description of the critical defects. In the standard approach, the failure probability $P_{f}$, for volume defects is given by $P_{f}=1-\exp \left[-(1 / m !)^{m}\left(V / V_{u}\right)\left(S_{n} / S_{u}\right)^{m} I(V)\right]$ where $V_{u}$ and $S_{u}$ denote the volume and stress of a reference, $S_{n}$ is the nominal stress on the material and I(V) is the stressvolume integral (the parameter $m$ is the socalled Weibull modulus). Leaving aside how one estimates experimentally all the parameters in the model, L. Dortmans and G. de With* (Centre for Technical Ceramics, Eindhoven *and Philips Research Laboratories) pointed out in their presentation there still remains the problem of applying data for a uniaxial test to a multiaxially stressed component. To do this the transformation stress-volume integral (or stress-surface integral if surface defects are critical) must be known. It has been shown [1] that analytical formulae for these integrals can be used for a simple one-dimensional bend test as they yield results accurate to $1 \%$, when compared with integrals calculated numerically by finite elements.

The problem is that one does not know how to apply these formulae to a multiaxial stress state. Several models have been proposed based upon different criteria for the
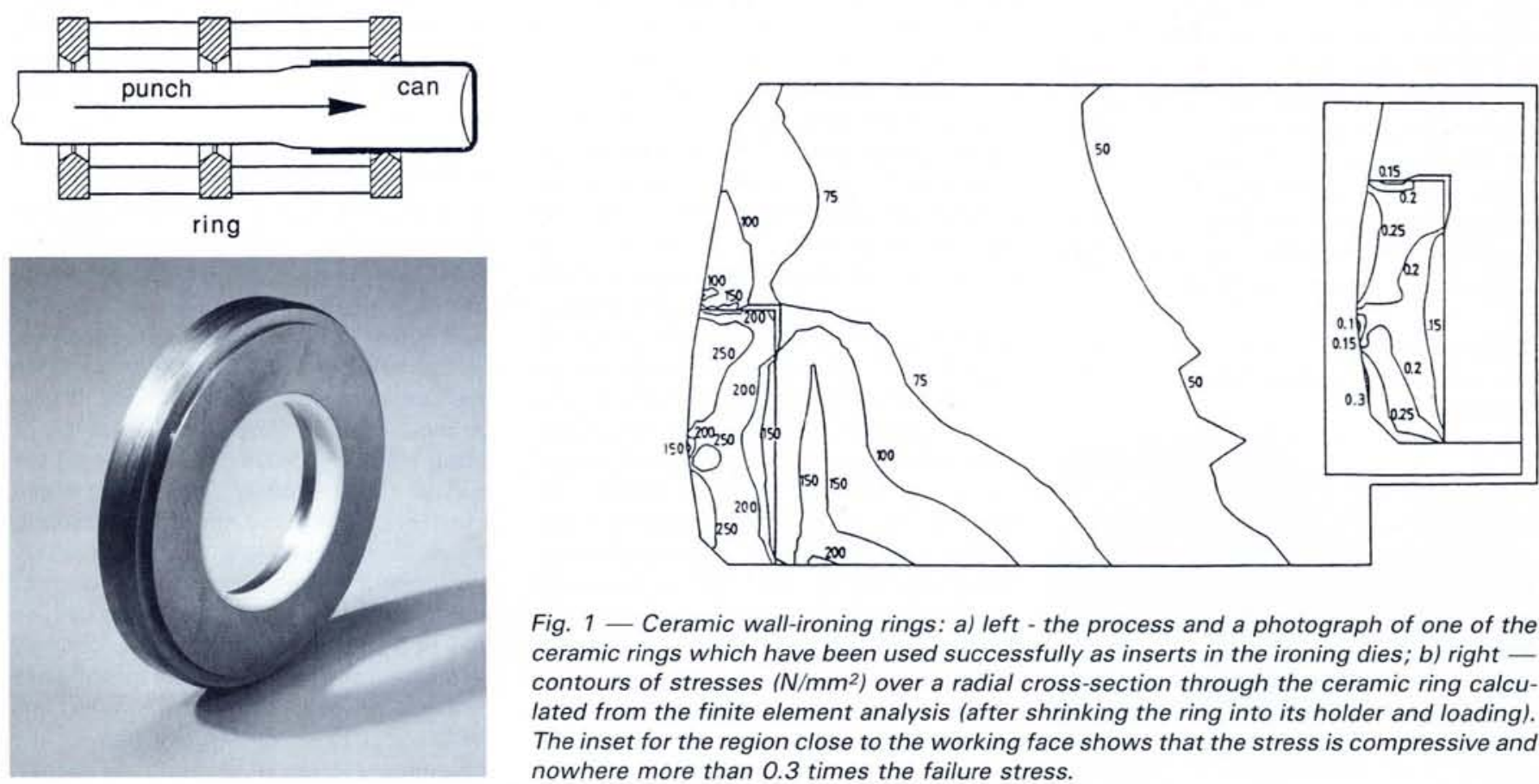

Fig. 1 - Ceramic wall-ironing rings: a) left - the process and a photograph of one of the ceramic rings which have been used successfully as inserts in the ironing dies; b) right contours of stresses $\left(\mathrm{N} / \mathrm{mm}^{2}\right)$ over a radial cross-section through the ceramic ring calculated from the finite element analysis (after shrinking the ring into its holder and loading). The inset for the region close to the working face shows that the stress is compressive and nowhere more than 0.3 times the failure stress. 